\title{
The Susceptibility of Leptinotarsa decemlineata (Say) (Coleoptera: Chrysomelidae) to Spinosad
}

\author{
Bahram Faridi $^{1}$, Ali Asghr Pourmirza ${ }^{1}$, Mohammad Hossien Safaralizadeh ${ }^{1}$ and \\ Mohammad Vali Taghaddosi ${ }^{2}$ \\ 1- Department of Entomology, Faculty of Agriculture, Urmia University, Urmia, \\ Iran \\ 2-Agricultural and Natural Resources Research Center of Zanjan \\ E-mail address: apourmirza1@yahoo.com
}

\section{ABSTRACT}

The susceptibility of Leptinotarsa decemlineata (Say) adults to spinosad was determined by leaf disk and glass jar techniques under laboratory conditions. The mortalities of the treated adults were recorded $72 \mathrm{~h}$ post treatments. $\mathrm{LC}_{50} \mathrm{~s}$ values in leaf disk and glass jar methods for adults were 98.50 and $108.34 \mathrm{mg}$ a.i/L, respectively. A significant linear relationship between $\mathrm{LC}_{50}$ values of individual population across test methods was detected $(\mathrm{r}=0.93, P<0.05)$. Both bioassay techniques were suitable for monitoring resistance to spinosad in Colorado potato beetle adult populations. Glass jar technique, however, exhibited less variability in $\mathrm{LC}_{50}$ estimates and showed a higher degree of sensitivity than the leaf disk method. Filter paper and leaf disk techniques for larvae were two bioassay methods used to determine spinosad susceptibility in L. decemlineata population. Both bioassay techniques exhibited a different level of susceptibility of the larvae to spinosad; however, the confidence interval values from filter paper method were narrow than the leaf disk assay technique. A significant direct relationship between $\mathrm{LC}_{50}$ values of individual population across test methods was observed $(\mathrm{r}=0.96, P<0.05)$. The glass jar and filter paper testing methods are simple and sensitive test techniques for measuring susceptibility of Colorado potato beetle adults and larvae to spinosad, respectively. The efficacy of spinosad can be conserved for a long period of time if it is judiciously rotated with other suitable insecticides in a spray program and the number of application is restricted.

Keywords: bioassay, Colorado potato beetle, leaf disk, paper disk, toxicity, spinosad

\section{INTRODUCTION}

Colorado potato beetle (CPB) is the most devastating defoliator of potato plants worldwide. Crop rotation and non-chemical tactics for regulating populations are useful in some situations; however, management of this pest has relied almost totally on chemical insecticides (Hare, 1990). Such reliance on insecticides for control has resulted in insecticide resistance to most insecticide chemical groups (Bishop \& Grafius, 1996; Stewart et al., 1997) and recently to commercialized new neonicotinoids (Mota-Sanchez et al., 2006). The Colorado potato beetle can evolve resistance very quickly to virtually every insecticide used against it in response to the local selection (Grafius, 1997).

Based on the previous reports it is inferred that to secure an acceptable level of CPB control, application of new insecticides with novel insecticidal mechanism of action is imperative. More recently the lack of spinosad interaction with known 
insecticidal target sites has been reported (Orr et al., 2009). Therefore, this insecticide with new mode of action could be an obvious candidate of choice in any CPB control program.

In Iran, no insecticides that effectively control CPB are registered. The current status of Colorado potato beetle resistance to conventional insecticide in Zanjan, an important potato-growing region of Iran, is unclear. Over the last decade, chemical insecticides mainly phosalone have been used successfully in many fields, but in many other cases the application of these compound has produced low mortality rates under field conditions. Concern about this irregular response and further development of resistance to conventional insecticides has made the search for insecticides with novel mechanism of action inevitable.

Recently the effects of spinosad on CPB have been reported in a number of cases (Elliott et al., 2007; Kowalska, 2008; Azimi et al., 2009). It has been repoted that spinosad with recommended rate has a limited impact on non-target organisms (Sarfraz et al., 2005).

Determining the baseline susceptibility levels and extent of resistance to insecticide in field populations of CPB is an essential first step toward developing its resistance management strategies (Olson et al., 2000). A baseline study also provides information on the natural variation of the concentration-mortality responses among populations.

Several bioassay methods for determining the resistance status of CPB to insecticides have been developed (Tisler \& Zehnder, 1990; Stewart et al., 1997; Zhao et al., 2000). The choice of appropriate exposure method can often improve discrimination between susceptible and resistant genotypes. Due to applied nature of this research, leaf disk and glass jar methods for adults, and filter paper and leaf disk techniques for larvae were used.

The current study was designed to determine the extent of L. decemlineata susceptibility to a commercial formulation of spinosad.

\section{MATERIAL AND METHODS}

\section{Insects and fields}

Colorado potato beetles were collected by hand from April to July 2009 from untreated potato fields in Zanjan, a town in Iran, and maintained under rearing conditions $\left(27 \pm 3^{\circ} \mathrm{C}, 50 \pm 10 \%\right.$ R.H. and a photoperiod of $16: 8 \mathrm{~L}$ : D). The fields were in a major agricultural area with high crop diversity. In region to control $L$. decemlineata, phosalone as a common insecticide was applied at least once per year. Adults.

For each test, depending on availability 150-200 first-generation of Colorado potato beetle adults was collected from untreated fields. The insects were returned to the laboratory and reared on greenhouse grown untreated fresh potato foliage inside plastic boxes with screen lids. The boxes were maintained under rearing conditions for two days before initiation of each test.

\section{Larvae}

During the period of oviposition by overwintered Colorado potato beetle adults, potato leaves containing egg masses were collected from sample sites. The samples were returned to the laboratory and placed on moist filter paper inside plastic cups and monitored every $24 \mathrm{~h}$ until hatch. Leaves with neonate larvae were placed on greenhouse grown untreated fresh potato foliage inside labeled plastic boxes with screen lids. Boxes were maintained under rearing conditions. 


\section{Insecticide}

A commercial formulation of Spinosad (Tracer ${ }^{\hat{A}}{ }^{\prime} \tilde{B}$ 24OSC) which was a gift from Dow AgroScience Inc, was used in all bioassays.

\section{Biossays}

For each developmental stage on the basis of preliminary tests, five concentrations of spinosad was tested to produce $\approx 25-75 \%$ mortality at the lowest and the highest concentrations, respectively (Robertson et al., 2007). In each trial, untreated control group was included. Fresh preparations of spinosad were made for each bioassay. At the time of bioassays adults and larvae were selected for uniformity in size and only vigorous insects were used. The treatments were replicated four times on four different days. In each bioassay method, results of all replicates were pooled.

\section{Adult studies}

In leaf tests, on the basis of preliminary tests five concentrations of spinosad $(30,60,100,130$ and $170 \mathrm{mg}$ a.i./L) and a control group were used in each test. Potato leaflets were excised from the middle to upper portion of green house grown plants and dipped in aqueous solutions with different concentrations of spinosad. Citowet was used as a surfactant to ascertain complete wetting of the leaves. After air-drying, the cut end of the stems were wrapped in nonabsorbent cotton wool and inserted in a $75 \mathrm{ml}$ glass vial filled with tap water. Then after the leaves were transferred to larger translucent boxes (about $270 \times 150 \times 90 \mathrm{~mm}$ ) which contained 20 randomly selected beetles. The boxes were sealed with tight fitting lids. The box lid had a $50 \mathrm{~mm}$ diameter hole in the center sealed with terylene net. The control group was provided with untreated potato leaves. The set ups were then kept in the rearing room under standard conditions. Mortality was recorded after $72 \mathrm{~h}$. Beetles were scored dead if no movement was observed after they were prodded with a hot dissecting needle. Test was replicated four times.

In glass jar tests, the bioassay procedure involves continuous exposure of insects within 500-ml glass jars coated with a known quantity of spinosad solutions. Based on preliminary trials, the five concentrations were 30,60,100, 130 and $170 \mathrm{mg}$ a.i./L. An Oxford pipette was used to apply $2.5 \mathrm{ml}$ of insecticide solution per glass jar. This amount was sufficient to cover the entire jar. Jar was rotated evenly in a hood until the water evaporated. Jar treated only with water served as a control treatment. Following treatment, the beetles were placed in jars ( 20 beetles per jar) and jars were sealed with screen lids and kept in environmental chamber at $27 \pm 3^{\circ} \mathrm{C}, 50 \pm 10 \%$ R.H. and 16:8 (L:D). Mortality was recorded after $72 \mathrm{~h}$. Beetles were considered dead if they did not move legs or antennae in response to probes. The trial was replicated four times.

\section{Larval studies}

In filter paper disk method, based on preliminary experiments five concentrations of spinosad viz, 20, 40, 80, 120 and $150 \mathrm{mg}$ a.i./L plus a control were used in each trial. Each filter paper disk (3-cm diameter) was dipped for $30 \mathrm{~s}$ in a known concentration of spinosad solution. Filter paper disk treated with water served as a control treatment. After air-drying, one each treated filter paper disk was placed into $35-\mathrm{mm}$ diameter plastic cup and moistened with $0.5 \mathrm{ml}$ distilled water. A camel's-hair brush was used to transfer 20 first instars larvae (up to $24 \mathrm{~h}$ old) from the stock culture to treated disk with minimum physical injury. Cups were covered with Parafilm and placed in environmental chamber. Mortality was recorded after $72 \mathrm{~h}$ post treatments. Those larvae that did not move when probed with a fine camel's-hair brush were considered dead. The trial was replicated four times. 
In leaf disk method, leaf disks (30-mm diameter) were cut from fully expanded greenhouse grown potato plants and dipped in five concentrations of spinosad $(20,40$, 80,120 and $150 \mathrm{mg}$ a.i./L) for $30 \mathrm{~s}$. Leaf disk treated with water served as a control treatment. After air-drying, each leaf disk was placed into $35-\mathrm{mm}$ diameter plastic cups containing $10 \mathrm{ml}$ of solidified $2 \%$ agar solution. Twenty first instars larvae (up to $24 \mathrm{~h}$ old) were placed into each plastic cup, sealed with Parafilm and incubated under rearing conditions. Mortality was recorded after $72 \mathrm{~h}$. Larvae were considered dead if they did not move when prodded with a fine brush. Bioassay was replicated four times.

\section{Data analysis}

Mortality data from all bioassays were analyzed with SPSS software (1999) assuming the probit model. Median lethal concentrations $\left(\mathrm{LC}_{50} \mathrm{~s}\right)$ and their corresponding $95 \%$ CI were estimated. The values and significance of $\chi^{2}$ and the $95 \%$ CI for potency ratios were determined (Norúsis, 2008). Parallel regression lines were also compared using overlapping confidence limits $(P \leq 0.05)$ of relative potencies as the criterion to detect significant differences in mortality. Pearson correlation was used to determine the correlation between $\mathrm{LC}_{50} \mathrm{~S}$ from two bioassay techniques.

\section{RESULTS}

\section{Adult studies}

A narrow range of variation in spinosad susceptibility existed between the bioassay techniques, as evidenced in $\mathrm{LC}_{50}$ values obtained in the bioassays (Table 1). Both the leaf disk and jar assays demonstrated spinosad susceptibility in the fieldcollected Colorado potato beetle populations. Comparisons across bioassays indicate that the $\mathrm{LC}_{50}$ value for population was higher in jar bioassay. For instance the $\mathrm{LC}_{50}$ of jar bioassay was 1.1 times more than the corresponding $\mathrm{LC}_{50}$ value in the leaf ingestion bioassay.

Table (1): Toxicity of spinosad to Leptinotarsa decemlineata first instars larvae and adults with different bioassay techniques $72 \mathrm{~h}$ after initiation of the trials

\begin{tabular}{|c|c|c|c|c|c|c|c|c|}
\hline \multirow{5}{*}{ Toxicity value } & \multicolumn{8}{|c|}{ Toxicity to life stages } \\
\hline & \multicolumn{4}{|c|}{ Larvae } & \multicolumn{4}{|c|}{ Adults } \\
\hline & \multirow{2}{*}{\multicolumn{2}{|c|}{ Paper disk }} & \multicolumn{2}{|c|}{ Leaf disk } & \multicolumn{2}{|l|}{ Jar } & \multicolumn{2}{|c|}{ Leaf } \\
\hline & & & \multicolumn{6}{|c|}{ Concentration $\mathrm{mg} /$ liter determined for } \\
\hline & $\mathrm{LD}_{50}$ & $\mathrm{LD}_{90}$ & $\mathrm{LD}_{50}$ & $\mathrm{LD}_{90}$ & $\mathrm{LD}_{50}$ & $\mathrm{LD}_{90}$ & $\mathrm{LD}_{50}$ & $\mathrm{LD}_{90}$ \\
\hline Lethal dose & 53.21 & 201.10 & 83.78 & 368.89 & 108.34 & 332.24 & 98.50 & 492.40 \\
\hline $\begin{array}{l}\text { Upper } \\
95 \% \text { CI }\end{array}$ & 61.50 & 287.70 & 100.68 & 653.87 & 123.59 & 488.31 & 118.05 & 977.50 \\
\hline Lower $95 \% \mathrm{CI}$ & 45.40 & 157.20 & 71.15 & 258.04 & 96.31 & 258.10 & 83.76 & 329.80 \\
\hline Slope \pm SE & $2.22 \pm 0.24$ & & $2.01 \pm 0.25$ & & $2.63 \pm 0.30$ & & $1.83 \pm 0.26$ & \\
\hline $\begin{array}{c}\text { Number of insects } \\
\text { tested }\end{array}$ & \multicolumn{2}{|l|}{480} & \multicolumn{2}{|c|}{480} & \multicolumn{2}{|l|}{480} & \multicolumn{2}{|c|}{480} \\
\hline$\chi^{2 \mathrm{a}}$ & \multicolumn{2}{|c|}{$4.59^{\mathrm{ns}}$} & \multicolumn{2}{|c|}{$4.73^{\mathrm{ns}}$} & \multicolumn{2}{|l|}{$2.48^{\mathrm{ns}}$} & \multicolumn{2}{|c|}{$3.10^{\mathrm{ns}}$} \\
\hline$P$ & \multirow[t]{2}{*}{0.20} & & \multirow{2}{*}{\multicolumn{2}{|c|}{$-0.81)$}} & \multirow[t]{2}{*}{0.48} & & \multirow{2}{*}{\multicolumn{2}{|c|}{0.38}} \\
\hline $\mathrm{RMP}_{50}{ }^{\mathrm{b}} \quad(95 \% \mathrm{CI})$ & & 0.65 & & & & $0.87(0$ & & \\
\hline
\end{tabular}

${ }^{a}$ Pearson's $\chi^{2}$ goodness-of-fit tests: all values of $P$ are $>0.05$ and the data fits regression model. ${ }^{b}$ Relative median potency estimate with lower and upper limits. 
Despite the differences in $\mathrm{LC}_{50} \mathrm{~S}$ of individual population across bioassay methods, the $\mathrm{LC}_{50} \mathrm{~S}$ from both bioassays were significantly correlated $(\mathrm{r}=0.94, P<$ $0.05)$.

This is an indication of that; both bioassay methods would be suitable for assessing magnitude of susceptibility to spinosad in field-collected CPB populations.

The slopes of the dose-response lines were relatively steep. This implies the homogeneity of CPB population from genetically stand point of view. That is, the application of high doses of spinosad could be a critical issue in selection more tolerant individual in immediate future. Estimation of relative median potency test demonstrated a similar toxicity of spinosad in both bioassay techniques (Table 1). Parallelism test of mortality-response lines yielded $\chi^{2}$ and $P$ values 4.33 and 0.03 , respectively. That is, the lines were not parallel.

\section{Larval studies}

$\mathrm{LC}_{50}$ values calculated from the leaf disk and filter paper disk techniques indicated a different pattern of susceptibility of larvae to spinosad from two bioassay techniques, as evidenced by non overlapping confidence intervals (Table 1). In leaf disk method, when spinosad was applied larvae were more than 1.57 times as resistant to spinosad compared with the paper disk technique. In filter paper disk method, variation in $\mathrm{LC}_{50} \mathrm{~S}$ confidence interval was narrow compared to those of leaf disk technique. Our data indicated that the filter paper bioassay method is superior to the leaf disk technique because there is visibly less variability in $\mathrm{LC}_{50}$ estimates obtained with the filter paper method compared with the leaf disk technique. Despite the differences in $\mathrm{LC}_{50} \mathrm{~S}$ of individual populations across bioassay methods, the $\mathrm{LC}_{50} \mathrm{~S}$ from both bioassays were significantly correlated $(\mathrm{r}=0.95, p<0.05)$. That is, both bioassay methods are suitable for assessing the extent of resistance to spinosad in CPB populations. The slopes of the dose-response are relatively steep and mortality rates between the highest and lowest were low. This is an indication of homogeneity of the population in sample site. Therefore, steps should be taken to promote population stability through integration of all IPM program tools such as augmentation of susceptible individuals in population. Parallelism test revealed a non significant difference between concentration-response lines $\left(\chi^{2}=0.27 ; P=0.60\right)$. This could be an indication of target site(s) similarity for two different exposure methods. Estimation of relative median potency yielded $0.65,0.50$, and 0.81 values for estimate, lower and upper confidence intervals, respectively. Spinosad, therefore, exerted different levels of toxicity to the larvae in different bioassay techniques.

\section{DISCUSSION}

A low level of variation in susceptibility to spinosad existed across geographically discrete samples of Colorado potato beetle. The underlying cause of this difference in susceptibility was not well understood but might be related to natural variation between the populations. However, in some instances it has been possible to associate lower levels of susceptibility with populations that were previously exposed to phosalone. Jar test rely on tarsal contact of adult beetles with insecticide deposits, and is sufficiently sensitive to determine the magnitude of resistance because it produces small confidence interval compared to leaf test.

The filter paper disk bioassay method demonstrated a narrow range of variation in the susceptibility of CPB larvae to spinosad between populations and the $\mathrm{LC}_{50}$ values were less than the leaf disk technique. Our results agreed with those reported by other scholars, i.e., filter paper test method have been successful in detecting more 
accurate insecticide resistance with insecticides (Pourmirza, 2005; Tisler \& Zehnder, 1990). The technique suggested a narrow range of variation in confidence intervals in present study with spinosad (45.4-61.5). This result suggests that the filter paper test method may be more efficient than the leaf disk test technique for evaluation resistance status of $L$. decemlineata larvae.

It has been reported that, different bioassay methods have led to different conclusions about the magnitude of resistance to insecticides on insects. For example, Scharf et al. (1995) used three bioassay methods to determine the extent of resistance of German cockroach to insecticides. They reported that the magnitude of resistance varied widely across bioassay techniques. Our results were different from this conclusion and showed that, despite the differences in $\mathrm{LC}_{50} \mathrm{~S}$ of individual population across bioassay methods, the $\mathrm{LC}_{50} \mathrm{~s}$ from both bioassays were significantly correlated. This is an indication of that; both bioassay methods in either case would be suitable for assessing magnitude of susceptibility to insecticide in field-collected CPB populations.

Based on the estimated $\mathrm{LC}_{90}$ values, the toxicity of the spinosad tested could be rated in the following order: paper disk $>$ glass jar $>$ larval leaf disk $>$ adult leaf disk. This implies that, paper disk and glass jar techniques are appropriate bioassay methods at the discrimination concentration level. There is general agreement that, an ideal bioassay method is one that is fast, labor efficient, with acceptable precision. According to these criteria, jar test technique for adults and the filter paper test method for larvae can produce more accurate $\mathrm{LC}_{50}$ and $\mathrm{LC}_{90}$ values when assessing spinosad susceptibility in field-collected CPB populations.

It has been stated that CPB is not resistance to spinosad and this compound has medium residual (10-14 days) activity to the pest (Ragsdale et al., 2007). The medium residual efficacy of spinosad and the need for contact with or ingestion of the chemical are indications of necessity for good coverage. It is inferred that targeting the two seasonal spinosad sprays at first generation CPB could improve damage control. Comparisons with the toxicity of phosalone determined in identical experiments (Pourmirza, 2005) were made. The $\mathrm{LD}_{50}$ s range obtained in that study for phosalone against five populations of Colorado potato beetle adults and larvae were $48.72-64.12 \mathrm{mg}$ ai/L. Apparently spinosad is somewhat equally toxic to insects exposed in the current trials is phosalone. In terms of efficacy, it seems more likely that the efficacy of spinosad could be conserved for a long period of time, if it is judiciously rotated with other suitable insecticides in a spray program and the number of application is restricted to two sprays at first generation.

\section{ACKNOWLEDGMENTS}

The authors are grateful to vice president of research of Urmia University for financial support. Dow AgroSciences-Iran for kindly providing spinosad as a gift.

\section{REFERENCES}

Azimi, M., Pourmirza, A.A., Safaralizadeh, M.H. and Mohitazar, M.V. (2009). Studies on the lethal effects of spinosad on adults of Leptinotarsa decemlineata (Say) (Coleoptera: Chrysomelidae) with two bioassay methods. Asian Journal of Biological Sciences, 2: 1-6. 
Bishop, B.A. and Grafius, E.J. (1996). Insecticide resistance in the Colorado potato beetle. In: Jolive PH, Cox ML (eds) Chrysomelidae biology, pp.355-377. SPB Academic, Amsterdam.

Elliott, R., Bejamin, M. and Gillott, C. (2007). Laboratory studies of the toxicity of spinosad and deltamethrin to Phyllotreta cruciferae (Coleoptera: Chrysomelidae). Canadian Entomologist, 139: 534-544.

Grafius, E.J. (1997). Economic impact of insecticide resistance in the Colorado potato beetle on the Michigan potato industry. Journal of Economic Entomology, 90: 1144-1151.

Hare, J.D. (1990). Ecology and management of the Colorado potato beetle. Annual Review of Entomology, 35: 81-100.

Kowalska, J. (2008). Laboratory studies of the activity of spinosad against Leptinotarsa decemlineata (Say) depending on different temperature. In: $16^{\text {th }}$ IFOAM World Congress, Modena, Italy, June 16-20, 2008. Archived at http://orgprints.org/view/projects/conference.html.

Mota-Sanchez, D., Hollingworth, R.M., Grafius, E.J. and Moyer, D.D. (2006). Resistance and cross-resistance to neonicotinoid insecticides and spinosad in the Colorado potato beetle, Leptinitarsa decemlineata (Say) (Coleoptera: Chrysomelidae). Pest Management Sciences, 62: 30-37.

Norusis, M.J. (2008). SPSS 16.0 Guide to Data Analysis. Upper Saddle River, N.J.: Prentice Hall, New York.

Olson, E. R., Dively, G.P. and Nelson, J.O. (2000). Baseline susceptibility to imidacloprid and cross-resistance patterns in Colorado potato beetle (Coleoptera: Chrysomelidae) populations. Journal Economic Entomology, 93: 447-458.

Orr, N., Shaffner, A.J., Richey, K. and Crouse, G.D. (2009). Novel mode of action of spinosad was determined against adult of Leptinotarsa decemlineata. Cpedia. Com/wiki?

Pourmirza, A.A. (2005). Local variation in susceptibility of Colorado potato beetle (Coleoptera: Chrysomelidae) to insecticides. Journal of Economic Entomology, 98: 2176-2180.

Ragsdale, D., Radcliffe, E.B., Burkness, S. and Hahn, J. (2007). Colorado potato beetles in home gardens. University of Minnesota Extension. http://134.84.92.130/distribution/horticulture/M1200.html.

Robertson, J.L., Russell, R.M., Preisler, H.K. and Savin, E. (2007). Pesticide Bioassays with Arthropods, CRC, Boca Raton, FL.

Sarfraz, M., Dosdall, L.M. and Keddie, B.A. (2005). Spinosad: a promising tool for integrated pest management. Outlooks on Pest Management, 16: 78-84.

Scharf, M.E., Bennett, G.W., Reid, L.B. and Qui, C. (1995). Comparison of three insecticide detection methods for the German cockroach (Dictyoptera: Blattellidae). Journal of Economic Entomology, 88: 536-542.

SPSS, (1999). SPSS User's Mannual. Release 10.0 SPSS, Chicago, IL.

Stewart, J.G., Kennedy, G.G. and Sturz, A.V. (1997). Incidence of insecticide resistance in populations of Colorado potato beetle, Leptinotarsa decemlineata (Say) (Coleoptera: Chrysomelidae), on Prince Edward Island. Canadian Entomologist, 129: 21-26.

Tisler, A.M. and Zehnder, G.W. (1990). Insecticide resistance in the Colorado potato beetle (Coleoptera: Chrysomelidae) on the Eastern Shore of Virginia. Journal of Economic Entomology, 83: 666-671.

Zhao, J.Z., Bishop, B.A. and Grafius, E.J. (2000). Inheritance and synergism of resistance to imidacloprid in the Colorado potato beetle (Coleoptera: Chrysomelidae). Journal of Economic Entomology, 93: 1508-1514. 